\title{
Progresso de Epidemias e Avaliação de Danos da Ferrugem em Clones de Álamo
}

\author{
Louise L. May-De Mio ${ }^{1}$, Lilian Amorim² \& Luciene M. Moreira ${ }^{1}$ \\ ${ }^{1}$ Departamento de Fitotecnia e Fitossanitarismo, Setor de Ciências Agrárias, Universidade Federal do Paraná, \\ Cx. Postal 19061, CEP 80035-050, Curitiba, PR, e-mail:maydemio@ufpr.br; \\ ${ }^{2}$ Departamento de Entomologia, Fitopatologia e Zoologia Agrícola, ESALQ, USP, Cx. Postal 9, \\ CEP 13418-900, Piracicaba, SP
}

(Aceito para publicação em 16/05/2006)

Autor para correspondência: Louise Larissa May-De Mio

MAY-DE MIO, L.L., AMORIM, L. \& MOREIRA, L.M. Progresso de epidemias e avaliação de danos da ferrugem em clones de álamo. Fitopatologia Brasileira 31:133-139. 2006.

\section{RESUMO}

A ferrugem do álamo (Melampsora medusae) causa sérios prejuízos no viveiro e tem sido cada vez mais freqüente em plantações, principalmente nos clones mais suscetíveis. O comportamento dos clones em relação a epidemias de ferrugem nas plantações brasileiras não é conhecido. Este trabalho teve como objetivos quantificar o progresso de epidemias em sete clones de álamo e correlacionar doença com produção por meio de relações entre incidência, severidade e índice de área foliar com o diâmetro do tronco do hospedeiro. As avaliações foram realizadas em sete clones de álamo: 'Argos’; 'Gaupiara'; 'Guarani'; 'JB'; 'Latorre'; 'SJ'; 'SM'. Cada clone compunha um talhão, todos com seis anos de idade. A severidade e a incidência da doença, o índice de área foliar (IAF) e o diâmetro à altura do peito (DAP) foram quantificados em dez plantas escolhidas aleatoriamente dentro de cada talhão de novembro a abril de 98/99, 99/00 e 00/01. Os clones mais suscetíveis à doença foram Latorre e Guarani, SJ foi resistente e Guapiara, SM, JB e Argos, intermediários. O modelo logístico apresentou um bom ajuste aos dados de severidade da ferrugem e a epidemia foi mais severa no ciclo de 99/00, onde as temperaturas variaram entre 15,5 e $26,5^{\circ} \mathrm{C}$. Não houve relação significativa entre severidade da doença e diâmetro à altura do peito (DAP). Nos clones suscetíveis, a duração da área foliar (DAF), que é a integralização da curva do IAF, foi relacionada com o DAP com coeficientes de determinação da regressão linear de 0,73 e 0,56 para Guarani e Latorre, respectivamente. O clone Latorre mostrou-se relativamente tolerante à doença, com alta severidade (100 a 150 pústulas/ $\mathrm{cm}^{2}$ ) e redução do DAP de 5\%, enquanto no Guarani a redução do DAP foi de $17 \%$.

Palavras-chave adicionais: Populus spp., resistência varietal.

\begin{abstract}
Progress of epidemics and assessment of damage caused by rust in poplar clones

Poplar rust (Melampsora medusae) causes serious damage in nurseries and has been frequent in commercial fields, mainly in susceptible clones. The behavior of the clones in the field in relation to this disease is as yet unknown in Brazil. The aims of this study were to quantify the disease's progress in the field in seven poplar clones and to correlate disease with production using incidence, severity, leaf area index (LAI) and diameter at breast height of a man (DBH). Evaluations were made monthly, from November to April, during the 98/99, 99/00 and 00/01 cycles. Disease incidence and severity, leaf area duration (LAD- integralization of LAI), and diameter at breast height (DBH) were assessed in clones 'Argos', 'Gaupiara', 'Guarani', 'JB', 'Latorre', 'SJ' and 'SM'. The most susceptible clones were Latorre and Guarani, clone SJ was resistant and Guapara, SM, JB and Argos were moderately susceptible. The logistical model showed a good fit to disease severity in the three cycles assessed. The epidemic was more severe in the 99/00 cycle when the temperature varied from 26.5 to $15.5^{\circ} \mathrm{C}$. No significant correlation was obtained between disease severity and DBH. In the susceptible clones, the LAD was related to DBH and the coefficient of determination in linear regression was 0.73 and 0.56 in Guarani and Latorre, respectively. The latter clone was tolerant to disease; the percentage of DBH reduction was only $5 \%$, while the clone Guarani showed $17 \%$ of reduction of $\mathrm{DBH}$ in relation to treated plants.
\end{abstract}

Additional keywords: Populus spp., varietal resistance.

\section{INTRODUÇÃO}

No vale do rio Iguaçu, Estado do Paraná, vários híbridos de álamo (Populus spp.) são utilizados para produção de madeira, principalmente por indústrias de palito de fósforo. A maioria do material genético inclui a espécie Populus deltoides Bartram ex Marshall suscetível à ferrugem das folhas (Melampsora medusae Thüm.) (Spiers, 1975; 
McCracken et al., 1984; Newcombe \& Chastagner, 1993). No Brasil, pouco se sabe sobre o comportamento dessa doença em plantações, apesar de seu relato remontar a 1945 em São Paulo (Viégas, 1945) e a 1968 no Paraná (Nowacki \& Fontoura, 1968). Os sintomas são pústulas diminutas de coloração amarela a alaranjada em ambas as superfícies foliares. Quando as pústulas coalescem, formam-se regiões necrosadas, levando à queda prematura das folhas.

A ferrugem do álamo pode ser causada por diferentes espécies do gênero Melampsora, como M. laricis-populina Kleb., M. allii-populina Kleb. e M. medusae, entre outras. No Brasil, apenas a espécie $M$. medusae foi relatada em plantas de Populus. A ferrugem causada por M. medusae é macrocíclica e heteróica, tendo como hospedeira alterna várias gimnospermas, inclusive Larix (Walker, 1975). Ainda não há relatos de ocorrência de $M$. medusae em hospedeiras alternas no Brasil. É possível que a sobrevivência do patógeno ocorra em plantações brasileiras pela continuidade de ciclos secundários no próprio álamo, pois, apesar do hábito caducifólio, algumas plantas mantêm as folhas por praticamente todo o ano. Esta forma de sobrevivência já foi reportada em países de clima temperado, quando da ocorrência de invernos amenos (Chastagner, 2001).

A desfolha prematura causada por M. medusae provoca redução de $30 \%$ a $65 \%$ no volume da planta no viveiro (Widin \& Schipper 1976; Steenackers et al., 1995; May-de Mio et al., 2002). Trabalhos realizados na França relacionaram o efeito da espécie $M$. laricis-populina com a qualidade de madeira, mostrando que a desfolha provocada por uma epidemia severa reduz o crescimento da planta no ano seguinte $\mathrm{e}$, em conseqüência, o cerne não se forma completamente (Steenachers et al., 1995).

O controle da doença por meio da pulverização de fungicidas, tanto no viveiro como na plantação, embora eficiente, não é a medida usual de controle nos grandes países produtores (Giorcelli \& Vietto, 1995; Pandey et al., 1996). No Brasil, no entanto, o controle tem sido feito rotineiramente por meio de pulverizações aéreas de triazóis, apesar do alto custo envolvido na operação e da possível agressão ao meio ambiente. O plantio de clones resistentes à ferrugem é a medida de controle adotada por vários países produtores de álamo, embora sua eficácia seja reduzida em virtude da alta variabilidade do patógeno (Moltzan, 1991; Newcombe et al., 1996). Os plantios comerciais de álamo no Estado do Paraná são recentes e as pesquisas visando resistência a doenças incipientes.

Para avaliar a eficiência de medidas de controle à ferrugem, sejam elas baseadas em controle químico ou genético, é importante quantificar não apenas as variáveis relacionadas à intensidade da doença como severidade, mas também a desfolha. A queda de folhas em decorrência da elevada severidade faz com que a intensidade da doença seja subestimada ao final do ciclo da cultura, pois as folhas remanescentes na árvore apresentam severidade mais baixa que as já caídas (Hamelin et al., 1993). Um dos fatores que contribui para que a desfolha seja desconsiderada na avaliação da doença é a dificuldade embutida em sua quantificação, notadamente em árvores de grande porte. A quantificação da desfolha depende da coleta freqüente de folhas caídas sob a copa. Em virtude da grande altura das árvores, a avaliação é imprecisa, haja vista a deriva das folhas pelo vento. Como medida alternativa, pesquisadores recorreram à avaliação do índice de área foliar (IAF) como indicativo indireto da desfolha. A quantificação do IAF pode ser feita com elevada precisão por meio de ceptômetros, equipamentos produzidos exclusivamente para esse fim, que consideram a área foliar que está na planta em função da luminosidade que atravessa o dossel.

Foram objetivos deste trabalho quantificar o progresso de epidemias de ferrugem do álamo em condições naturais de infecção no vale do Iguaçu, PR; avaliar a suscetibilidade de sete clones de álamo à doença; e correlacionar doença com a produção por meio de relações entre incidência, severidade e índice de área foliar com o diâmetro do tronco do hospedeiro.

\section{MATERIAL E MÉTODOS}

As avaliações foram realizadas em sete talhões da fazenda São Joaquim da empresa Andrade Latorre, localizada no município de São Mateus do Sul, PR, na região do vale do Iguaçu, plantadas com sete clones de álamo (Tabela 1). Cada talhão era composto de oito linhas de dez plantas de um determinado clone de álamo, no espaçamento de $8 \mathrm{x}$ $8 \mathrm{~m}$. No início dos experimentos, as árvores estavam com

TABELA 1 - Clones de álamo (Melampsora medusae) avaliados: nome, descrição e suscetibilidade a ferrugem ( $M$. medusae) em relação à desfolha. Os clones são utilizados nos plantios do Vale do rio Iguaçu-PR, pela Empresa Andrade Latorre S.A.

\begin{tabular}{lllc}
\hline \hline Clone* & Descrição & $\begin{array}{c}\text { Suscetibilidade } \\
\text { no viveiro** }\end{array}$ \\
\hline Argos & híbrido de & P. deltoides (americano) & baixa \\
Guapiara híbrido de & P. deltoides (americano) & média \\
Guarani & híbrido de & P. deltoides (americano) & médi \\
JB & híbrido de & P. deltoides (amcricano) & mćdia \\
Latorre & híbrido de & P. deltoides (americano) & alta \\
SJ & híbrido de & P. deltoides (euroamericano) & baixa \\
SM & híbrido de & P. deltoides (americano) & média \\
\hline *Clones trazidos da Argentina na década de 90, todos da secção Aigeiros. \\
** May-De Mio et al., 2002.
\end{tabular}

seis anos de idade e permaneceram isoladas de qualquer tratamento químico com fungicidas e sob infecção natural do patógeno. Dez plantas de cada talhão foram marcadas e numeradas para o monitoramento da doença, do índice de área foliar (IAF) e do diâmetro à altura do peito (DAP).

As avaliações do progresso da ferrugem iniciaram-se em novembro e estenderam-se até abril. Nas safras 98/99 e 99/00, as avaliações tiveram freqüência mensal e no ciclo 00/01 a freqüência foi irregular, mensal ou quinzenal. A cada data de avaliação determinou-se a incidência de folhas doentes e estimou-se a severidade da doença por meio 
do número médio de pústulas por centímetro quadrado em amostras de 100 folhas de cada clone. As folhas foram coletadas de dez árvores marcadas, sempre de dois ramos localizados no terço inferior e em lados opostos da árvore. Cinco folhas eram retiradas ao acaso do terço médio de cada ramo.

$\mathrm{O}$ enfolhamento das árvores durante o período de desenvolvimento das plantas foi avaliado pelo índice de área foliar, utilizando-se um ceptômetro (ACCUPAR DEACON). O equipamento possui uma barra de sensores, que medem a radiação fotossinteticamente ativa nos comprimentos de onda de 400 a $700 \mathrm{~nm}$, estimando assim, indiretamente, o IAF (Canteri, 1998). Neste caso, as avaliações foram feitas mensalmente, iniciando às 11:00 h da manhã. Para cada árvore tomava-se a medida da radiação em pleno sol e sob a projeção da copa, com a barra voltada para o tronco da árvore, com duas leituras/árvore. Além disso, foram feitas avaliações mensais do DAP das plantas marcadas de cada talhão.

Ao final do segundo e terceiro ano de avaliações, outras dez plantas de cada clone com a mesma idade e no mesmo talhão, as quais receberam tratamento no solo com o fungicida granulado a base de cyproconazole ( $40 \mathrm{~g}$ do produto comercial altomix/árvore em novembro de cada ano), foram também marcadas. Nestas plantas foram avaliados, no final da estação de crescimento (início de abril), o DAP e o IAF. Tais dados foram utilizados para determinar a porcentagem de redução de DAP de cada clone, comparativamente entre plantas tratadas e não tratadas.

\section{Análise dos resultados}

O modelo logístico, $y=b_{1} /\left(1+b_{2}{ }^{*} \exp \left(-b_{3}{ }^{*} x\right)\right)$, onde $y$ representa a severidade, $b_{1}$ corresponde à estimativa da assíntota máxima, $b_{2}$ é um parâmetro relacionado com o inóculo inicial, $b_{3}$ corresponde à taxa de crescimento da doença e $x$ é o tempo em dias, foi ajustado à curva de progresso da severidade de ferrugem para cada clone, em cada ano. Os ajustes foram feitos por meio de regressões nãolineares, utilizando o programa STATISTICA para Windows versão 5.0 (Statsoft, Tulsa, USA). Calculou-se também a área abaixo da curva de progresso da doença (AACPD) pelo método da integração trapezoidal (Berger, 1988). O IAF de cada planta ao longo do tempo para os sete clones foi integralizado obtendo-se valores de duração da área foliar (DAF), também por integração trapezoidal. Para avaliar o efeito da ferrugem no crescimento da planta foram feitas regressões lineares entre variáveis da doença (severidade máxima, AACPD e DAF) com o diâmetro da árvore à altura do peito (DAP).

A comparação dos clones foi feita por meio de análise de variância para as variáveis AACPD, DAP e DAF. A comparação das médias foi realizada com o teste de Tukey a 5\% de significância com o uso do programa Plot-IT para Windows (Cientific Programming Enterprices, Haslett, MI).

\section{RESULTADOS E DISCUSSÃO}

A incidência da doença não serviu como variável discriminatória da resistência, pois rapidamente 100\% das folhas avaliadas apresentaram sintomas, independentemente do nível de resistência do clone, à exceção do clone SJ no qual a incidência máxima foi de 50\% (Figura 1). Os valores de severidade, por sua vez, foram muito diferentes em cada clone (Figura 2). O modelo logístico apresentou um bom ajuste aos dados de severidade nos três anos avaliados e o parâmetro $b_{1}$ do modelo pode ser usado como indicativo da suscetibilidade à ferrugem (Figura 2; Tabela 2). Não houve ajuste do modelo aos dados do clone SJ em razão de sua elevada resistência à doença (Figura 2). Para todos os clones, com exceção novamente do SJ, a epidemia foi mais severa na estação 99/00, quando a severidade atingiu os maiores níveis, chegando a valores superiores a 200 pústulas $/ \mathrm{cm}^{2}$ nos clones Latorre e Guarani (Figura 2). Nos demais clones a severidade permaneceu em torno de 100 pústulas $/ \mathrm{cm}^{2}$, enquanto no ano anterior e posterior foi pelo menos $40 \%$

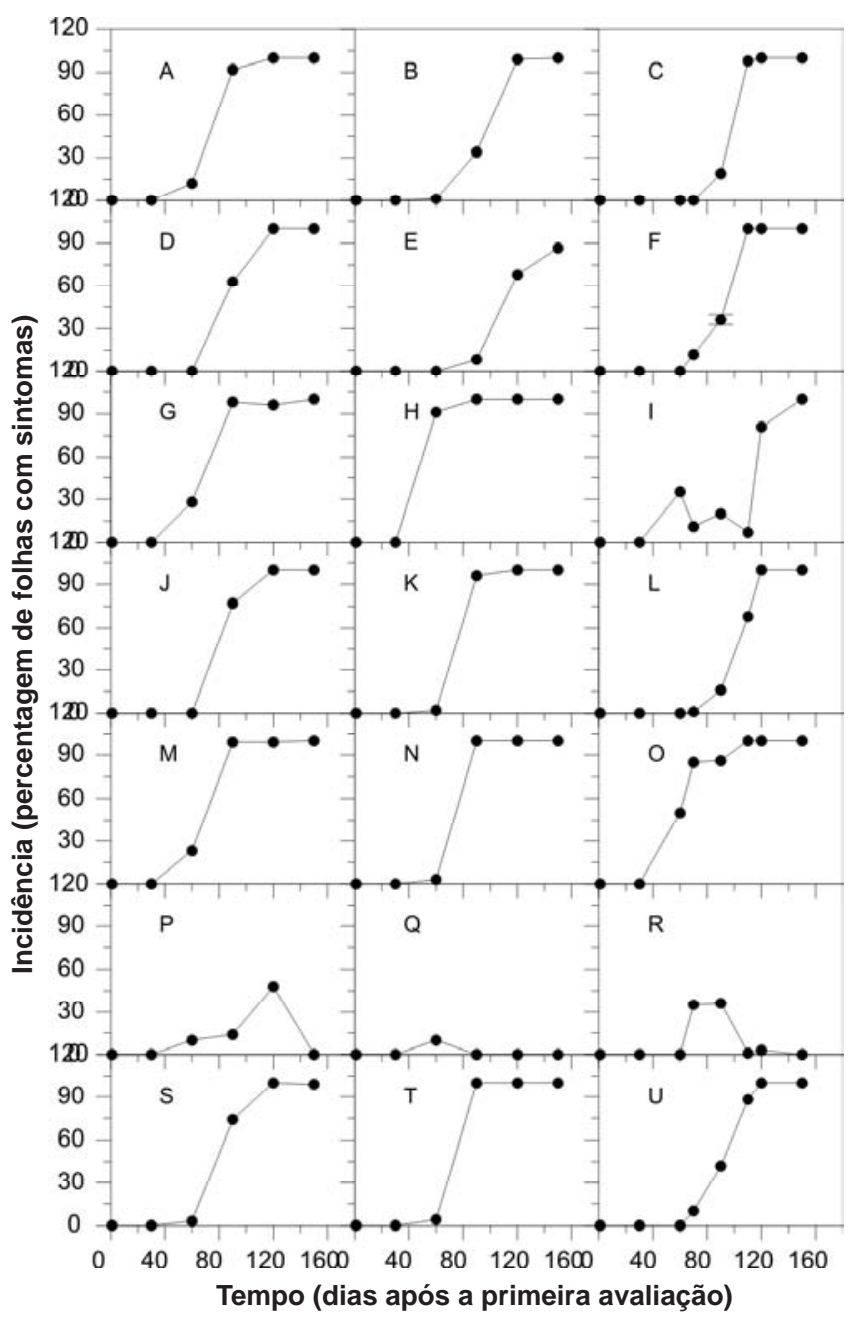

FIG. 1 - Incidência (porcentagem de folhas com pústulas) da ferrugem do álamo (Melampsora medusae) nos clones Argos (A, B, C), Guapiara (D, E, F), Guarani (G, H, I), JB (J, K, L), Latorre (M, N, O), SJ (P, Q, R) e SM (S, T, U) nos anos de 98/99 (coluna à esquerda), 99/00 (coluna central) e 00/01 (coluna à direita). As avaliações iniciaram-se em novembro de cada ano. 


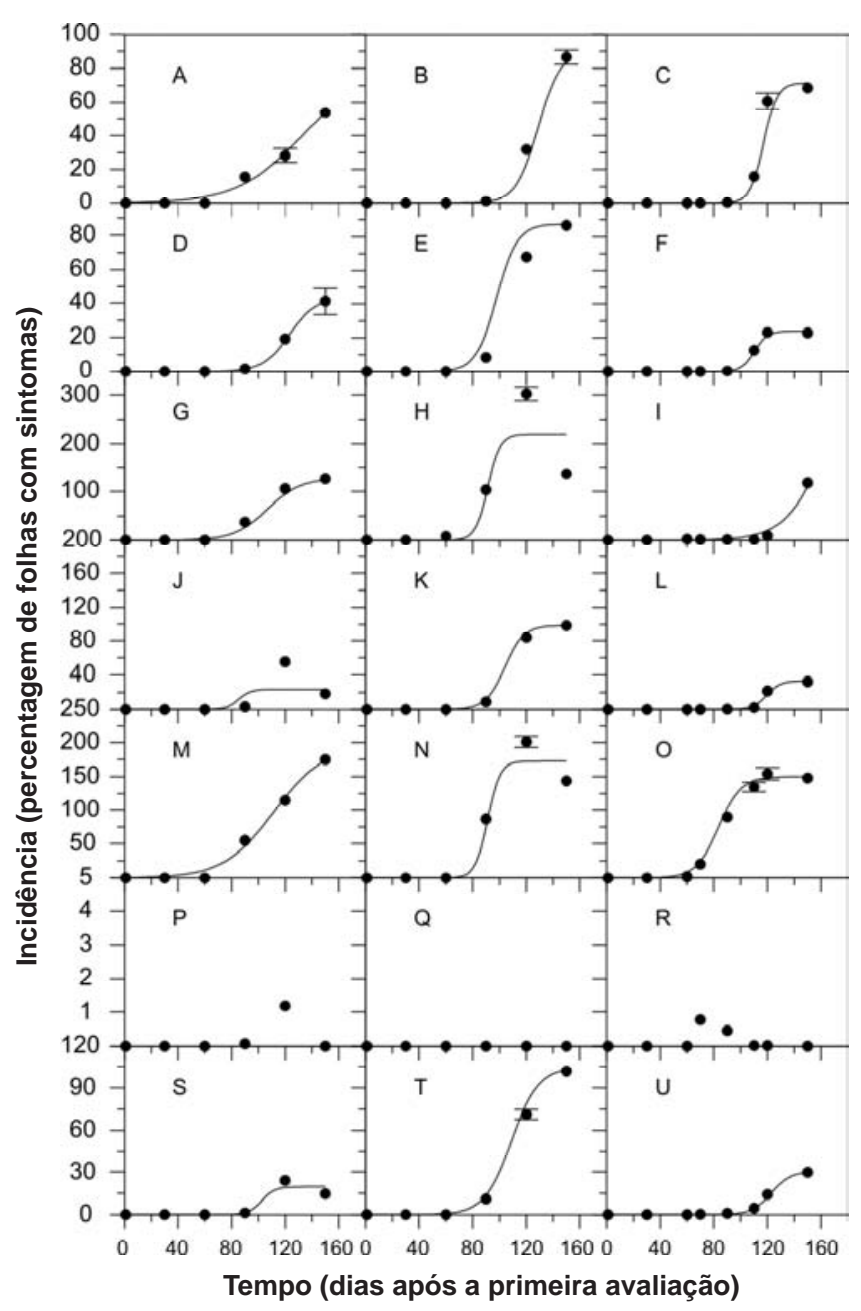

FIG. 2 - Severidade (número de pústulas/ $\mathrm{cm}^{2}$ ) da ferrugem do álamo (Melampsora medusae) nos clones Argos (A, B, C), Guapiara (D, E, F), Guarani (G, H, I), JB (J, K, L), Latorre (M, N, O), SJ (P, Q, R) e SM (S, T, U) nos anos de 98/99 (coluna à esquerda), 99/00 (coluna central) e 00/01 (coluna à direita). As avaliações iniciaramse em novembro de cada ano. Linhas indicam ajuste do modelo logístico aos dados (pontos).

inferior. A temperatura na safra 99/00 foi bastante favorável à ocorrência de epidemias da ferrugem do álamo no Paraná (May-De Mio \& Amorim, 2002). Nesse ciclo, a desfolha iniciada em janeiro foi progressiva até março, conforme mostram os valores de IAF do período (Figura 3). No ciclo 00/01 a epidemia foi tardia, embora a severidade final tenha sido semelhante à registrada em 98/99 (Figura 2). A desfolha nessa safra também foi tardia (Figura 3). Normalmente as árvores iniciam a desfolha natural a partir da segunda quinzena de abril. Essa queda antecipada das folhas, causada pela ferrugem, pode alterar a reserva de nutrientes para o inverno e provocar redução de crescimento da árvore no próximo ano.

O clone SJ, apesar de altamente resistente, apresentou uma queda acentuada do IAF, creditada à suscetibilidade do clone a outra doença foliar causada por Septoria musiva Peck, que também provoca desfolha (Figura 3). Em alguns casos observou-se um aumento no IAF na última avaliação
TABELA 2 - Coeficiente de determinação $\left(R^{2}\right)$ e parâmetros estimados pelo modelo logístico $y=b_{1} /\left[1+b_{2} * \exp \left(-b_{3} * x\right)\right]$, onde $y$ representa severidade, $x$, tempo, $b_{1}$ corresponde à assíntota máxima, $b_{2}$ está relacionado ao inóculo inicial e $b_{3}$ representa a taxa de progresso da doença, ajustado aos dados de progresso da ferrugem em diferentes clones de álamo (Melampsora medusae) nas safras 98/99, 99/00 e 2000/01

\begin{tabular}{|c|c|c|c|c|c|}
\hline \multirow{2}{*}{ Clones } & & \multicolumn{3}{|c|}{ Parâmetros do modelo logístico } & \multirow{2}{*}{$\mathbf{R}^{2}$} \\
\hline & & $\mathbf{b}_{1}$ & $\mathbf{b}_{2}$ & $\mathbf{b}_{3}$ & \\
\hline \multicolumn{6}{|c|}{ Latorre } \\
\hline$\bullet$ & $98 / 99$ & 195,92 & 398,89 & 0,05 & 0,99 \\
\hline$\bullet$ & $99 / 00$ & 172,74 & $5,13 \mathrm{e} 8$ & 0,22 & 0,98 \\
\hline$\bullet$ & $00 / 01$ & 150,25 & $2,28 \mathrm{e} 4$ & 0,12 & 0,99 \\
\hline \multicolumn{6}{|l|}{ Argos } \\
\hline$\bullet$ & $98 / 99$ & 79,65 & 223,25 & 0,04 & 0,98 \\
\hline$\bullet$ & $99 / 00$ & 90,47 & $5,25 \mathrm{e} 6$ & 0,12 & 0,99 \\
\hline$\bullet$ & $00 / 01$ & 71,42 & $1,50 \mathrm{e} 10$ & 0,20 & 0,99 \\
\hline \multicolumn{6}{|l|}{ JB } \\
\hline$\bullet$ & $98 / 99$ & 23,40 & $3,27 \mathrm{e} 8$ & 0,23 & 0,96 \\
\hline$\bullet$ & $99 / 00$ & 98,73 & $2,14 \mathrm{e} 6$ & 0,14 & 0,99 \\
\hline$\bullet$ & $00 / 01$ & 32,98 & $2,38 \mathrm{e} 10$ & 0,20 & 0,99 \\
\hline \multicolumn{6}{|l|}{ SM } \\
\hline$\bullet$ & $98 / 99$ & 19,45 & $2,32 \mathrm{e} 9$ & 0,21 & 0,95 \\
\hline$\bullet$ & $99 / 00$ & 104,48 & $5,42 \mathrm{e} 4$ & 0,10 & 0,99 \\
\hline$\bullet$ & $00 / 01$ & 30,24 & $3,05 \mathrm{e} 7$ & 0,14 & 0,99 \\
\hline \multicolumn{6}{|c|}{ Guarani } \\
\hline$\bullet$ & $98 / 99$ & 127,88 & $5,14 \mathrm{e} 3$ & 0,08 & 0,99 \\
\hline$\bullet$ & $99 / 00$ & 219,90 & $5,30 \mathrm{e} 8$ & 0,22 & 0,89 \\
\hline$\bullet$ & $00 / 01$ & 459,66 & $4,69 \mathrm{e} 4$ & 0,06 & 0,98 \\
\hline \multicolumn{6}{|c|}{ Guapiara } \\
\hline$\bullet$ & 98/99 & 43,68 & $2,27 \mathrm{e} 5$ & 0,10 & 0,99 \\
\hline$\bullet$ & $99 / 00$ & 87,45 & $3,32 \mathrm{e} 5$ & 0,13 & 0,99 \\
\hline - & $00 / 01$ & 23,99 & $1,13 \mathrm{e} 10$ & 0,21 & 0,99 \\
\hline
\end{tabular}

apesar da alta severidade da doença, devido a uma rebrota da árvore antes da desfolha de inverno (Figura 3). Este aumento ocorreu no ano de 99/00 quando a epidemia foi mais severa. Desfolhas repetidas, principalmente em árvores jovens em desenvolvimento, podem reduzir reservas nas raízes e predispor as árvores a outros patógenos (Schipper \& Dawson, 1974). Hamelin et al. (1993) observaram relação negativa significativa entre variáveis do progresso da doença e tempo de desfolha, o que confirma a interferência da doença na desfolha precoce.

As relações entre DAF e DAP não foram apresentadas para todos os clones avaliados. Apenas nos clones altamente suscetíveis, Guarani e Latorre, a regressão linear entre essas variáveis foi significativa em pelo menos um dos anos avaliados (Figura 4). A duração da área foliar sadia é uma variável que leva em conta a magnitude da área foliar e sua persistência no tempo, sendo conveniente no estabelecimento de relações com a produção (Watson, 1947). Assim, quanto maior a desfolha, menor a DAF e maior o impacto sobre a produção total de madeira, avaliada neste caso pelo DAP.

Não houve relação significativa entre as variáveis severidade máxima e DAP ou AACPD e DAP em qualquer 


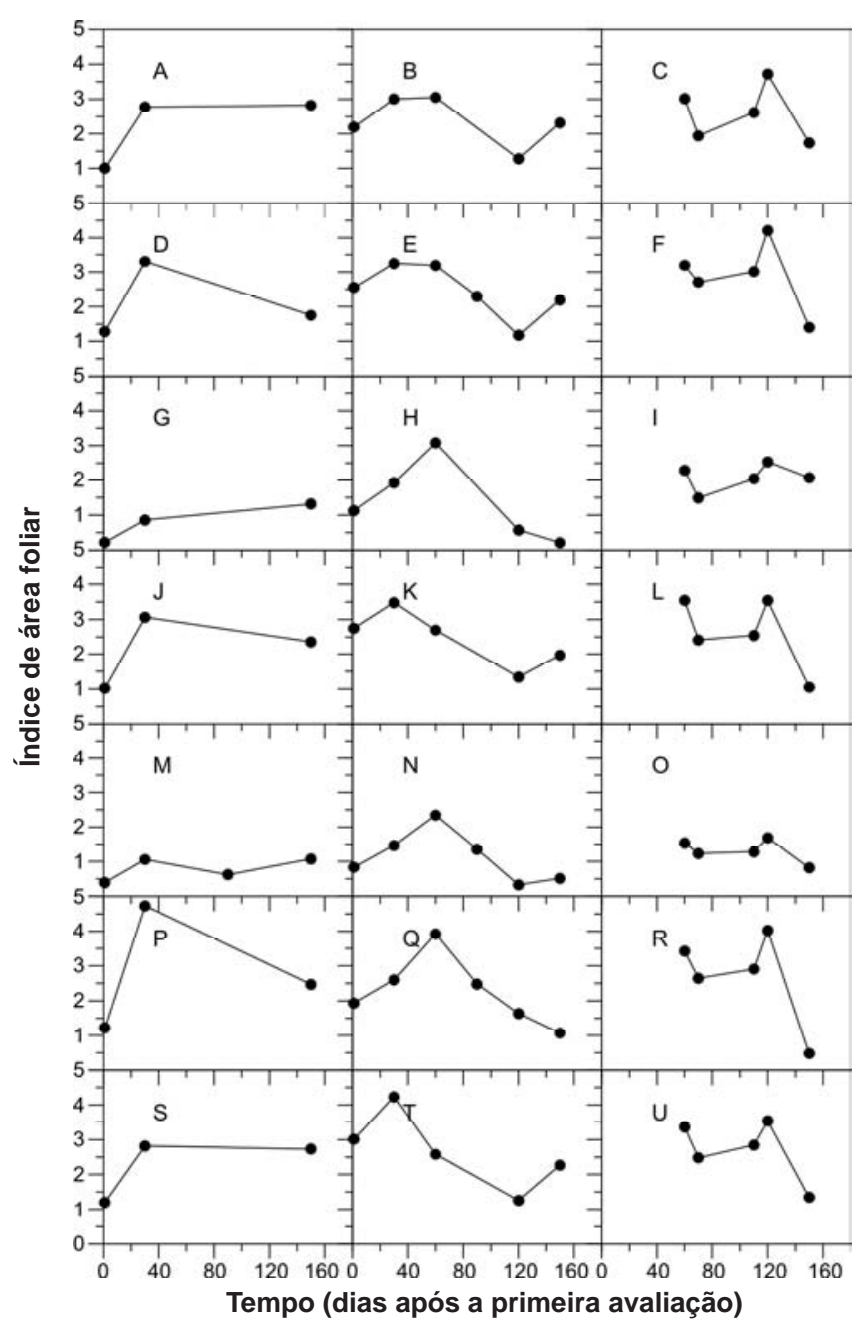

FIG. 3 - Índice de área foliar (IAF) nos clones de álamo (Melampsora medusae) Argos (A, B, C), Guapiara (D, E, F), Guarani (G, H, I), JB (J, K, L), Latorre (M, N, O), SJ (P, Q, R) e SM (S, T, U) nos anos de 98/99 (coluna à esquerda), 99/00 (coluna central) e 00/01 (coluna à direita). As avaliações iniciaram-se em novembro de cada ano.

dos clones analisados. A ausência de relação entre severidade e produção já foi relatada para mancha angular do feijoeiro (Bergamin Filho et al., 1997; Carneiro et al., 1997) e para ferrugem do feijoeiro, da aveia e do milho (Pataky et al., 1988; Bissonnette et al., 1994; Bergamin Filho \& Amorim, 1996). Uma das razões apresentadas para a falta de relação entre severidade e produção é a desfolha (Waggoner \& Berger, 1987). Nas doenças que provocam desfolha, a severidade média das folhas da planta é reduzida em razão da queda de folhas com severidade elevada. Conseqüentemente, a produção também é reduzida. Neste experimento, a produção foi correlacionada à severidade apenas na variedade Latorre quando dados de severidade de março de 2000 foram confrontados ao DAP de março de 2001 (Figura 5).

De acordo com a análise de variância da AACPD, realizada ano a ano, o clone Latorre foi classificado como suscetível; Guapiara, SM, Argos e JB como intermediários e SJ como resistente (Tabela 3).

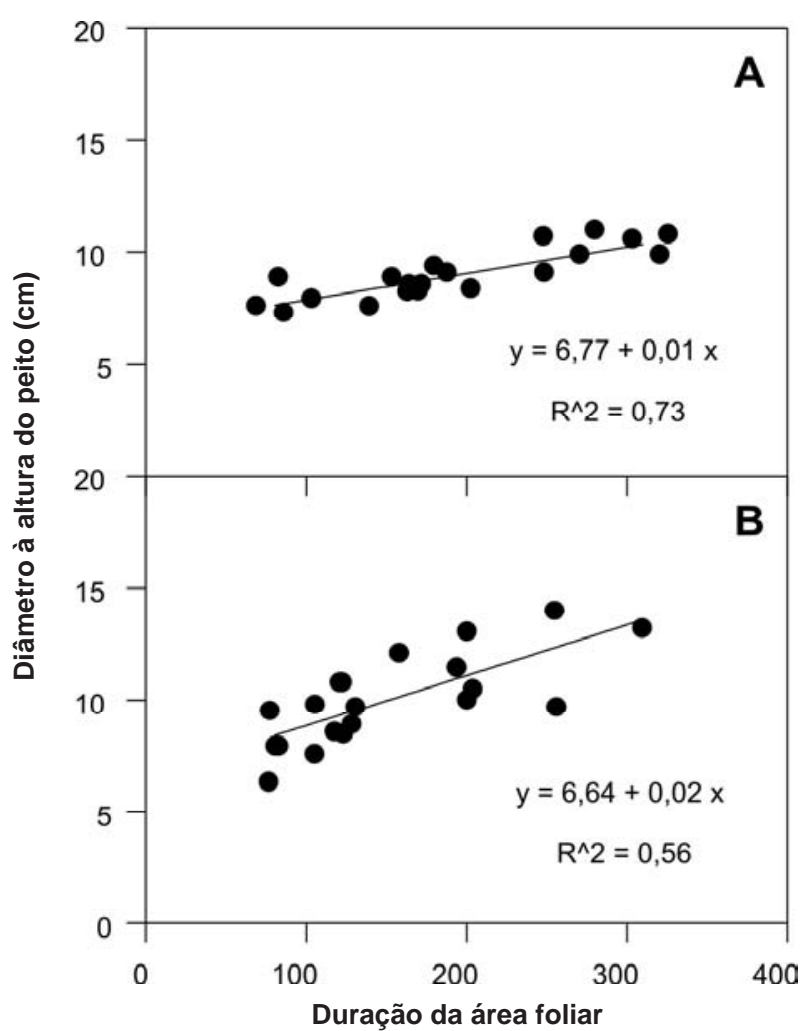

FIG. 4 - Relação entre diâmetro à altura do peito (DAP) e duração da área foliar (DAF) nos clones de álamo (Melampsora medusae) Guarani (A) e Latorre (B) nos anos de 98/99 e 99/00.

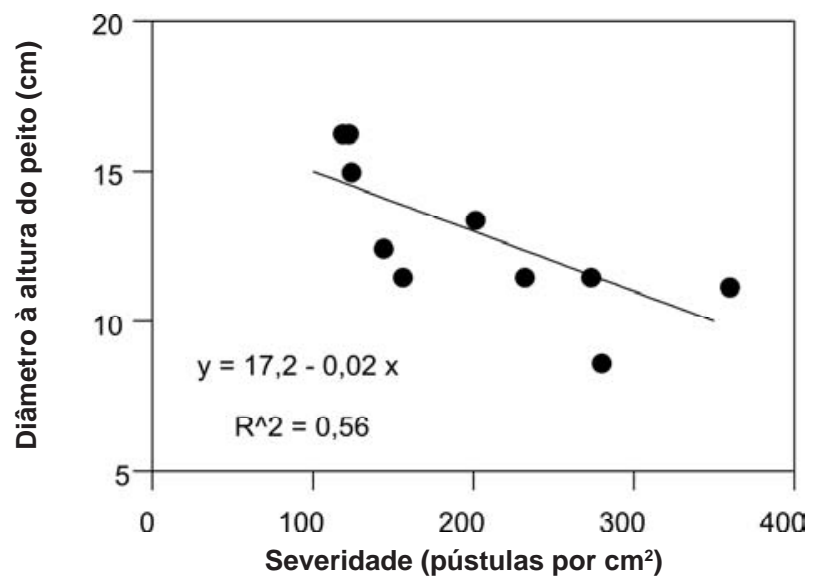

FIG. 5 - Relação entre diâmetro à altura do peito (DAP) em março de 2001 e severidade (número de pústulas de $/ \mathrm{cm}^{2}$ de folha) da ferrugem do álamo (Melampsora medusae) em março de 2000 no clone Latorre.

Com os dados coletados em 99/00 e 00/01 em parcelas tratadas com fungicidas granulados foi possível relacionar a redução de DAP com DAF e severidade (Figura 6). De acordo com as retas de regressão, os clones Latorre, SM, Guapiara e Argos apresentam tolerância em relação à doença e/ou à desfolha quando comparados com Guarani e 
TABELA 3 - Área abaixo da curva de progresso da severidade (AACPD) da ferrugem do álamo (Melampsora medusae), duração da área foliar (DAF) e diâmetro a altura do peito (DAP), avaliada em seis clones durante três anos consecutivos

\begin{tabular}{|c|c|c|c|c|c|c|c|c|c|}
\hline \multirow[b]{2}{*}{ Clones } & \multicolumn{3}{|c|}{ Avaliação de 98/99 } & \multicolumn{3}{|c|}{ Avaliação de 99/00 } & \multicolumn{3}{|c|}{ Avaliação de 00/01 } \\
\hline & $\begin{array}{l}\text { AACPD }{ }^{1} \\
\text { severidade }\end{array}$ & DAF & DAP & $\begin{array}{c}\text { AACPD }^{1} \\
\text { severidade }\end{array}$ & DAF & DAP & $\begin{array}{c}\text { AACPD }^{1} \\
\text { severidade }\end{array}$ & DAF & DAP \\
\hline SJ & $30,7 a^{2}$ & $476,8 \mathrm{~d}$ & $15,9 \mathrm{~cd}$ & $0 \mathrm{a}$ & $393,4 \mathrm{~b}$ & $16,7 \mathrm{c}$ & $13,4 \mathrm{a}$ & $259,4 \mathrm{~cd}$ & $17,9 \mathrm{bc}$ \\
\hline SM & $752,7 \mathrm{~b}$ & $356,7 \mathrm{bc}$ & $15,3 \mathrm{c}$ & $4558,2 \mathrm{c}$ & $415,6 \mathrm{~b}$ & $16,7 \mathrm{c}$ & $740,2 \mathrm{~b}$ & $255,5 \mathrm{~cd}$ & $18,5 \mathrm{~cd}$ \\
\hline Guapiara & $921,7 \mathrm{c}$ & $341,8 \mathrm{~b}$ & $15,6 \mathrm{c}$ & $4214,7 \mathrm{bc}$ & $395,5 \mathrm{~b}$ & $17,6 \mathrm{~cd}$ & $1154,6 \mathrm{bc}$ & $281,8 \mathrm{~d}$ & $19,2 \mathrm{~cd}$ \\
\hline JB & $1339,2 \mathrm{bc}$ & $350,6 \mathrm{~b}$ & $13,3 \mathrm{~b}$ & $4708,2 \mathrm{c}$ & $387,2 \mathrm{~b}$ & $14,6 \mathrm{~b}$ & $1033,7 \mathrm{bc}$ & $241,7 \mathrm{c}$ & $16,0 \mathrm{~b}$ \\
\hline Argos & $1963,4 \mathrm{c}$ & $354,3 \mathrm{~b}$ & $17,6 \mathrm{~d}$ & $2615,4 b$ & $377,1 \mathrm{~b}$ & $18,8 \mathrm{~d}$ & $2857,3 \mathrm{de}$ & $245,7 \mathrm{~cd}$ & $20,6 \mathrm{~d}$ \\
\hline Guarani & $4933,1 \mathrm{~d}$ & $130,0 \mathrm{a}$ & $8,2 \mathrm{a}$ & $9445,0 \mathrm{~d}$ & $256,4 \mathrm{a}$ & $9,9 \mathrm{a}$ & $2146,0 \mathrm{~cd}$ & $193,4 \mathrm{~b}$ & $12,8 \mathrm{a}$ \\
\hline Latorre & $5842,6 \mathrm{~d}$ & 108,6 a & $9,2 \mathrm{a}$ & $8140,6 \mathrm{~d}$ & $195,7 \mathrm{a}$ & $10,9 \mathrm{a}$ & $8411,2 \mathrm{f}$ & $123,5 \mathrm{a}$ & $12,7 \mathrm{a}$ \\
\hline $\begin{array}{l}\text { Coeficiente } \\
\text { Variação (\%) }\end{array}$ & 17,30 & 14,45 & 10,49 & 20,00 & 14,22 & 10,05 & 25,41 & 12,10 & 9,73 \\
\hline
\end{tabular}

${ }^{1}$ Para efeito da análise estatística os dados foram transformados em raiz $(\mathrm{x}+1)$, as médias apresentadas são as originais.

${ }^{2}$ Médias seguidas da mesma letra na coluna não diferem entre si pelo teste Tukey a $5 \%$ de significância.

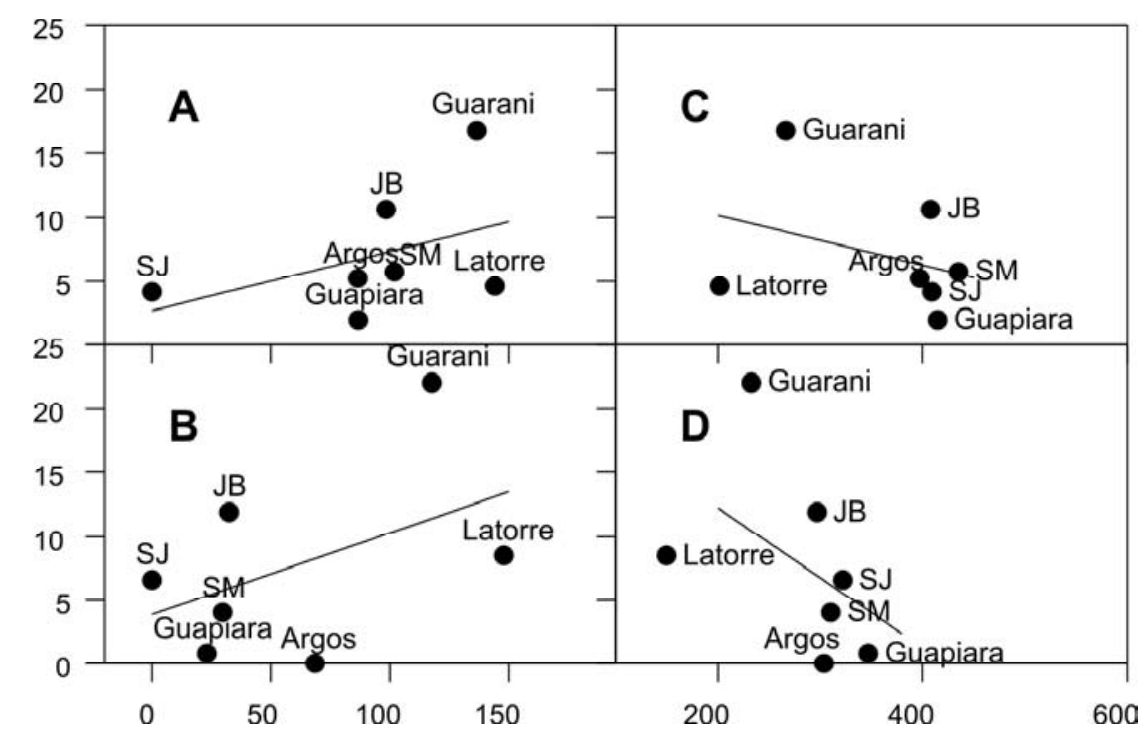

FIG. 6 - Redução do diâmetro a altura do peito (DAP) de sete clones de álamo (Melampsora medusae) expostos à infecção natural de Álamo em comparação a plantas tratadas com fungicida, em função da severidade final (número de pústulas $/ \mathrm{cm}^{2}$ em março) e duração da área foliar (DAF) nos ciclos de 99/00 (A, C) e 00/01 (B, D).

JB. Apesar do clone Latorre mostrar maior suscetibilidade que Guarani, sua tolerância à doença e à desfolha o tornam interessante para o plantio, pois a redução no DAP foi muito aquém da média dos outros clones (Figura 6). Os clones Guapiara, SM e Argos são também indicados pela moderada suscetibilidade e pela tolerância à doença.

Para maximizar o potencial de crescimento da árvore são necessários programas contínuos de seleção de clones resistentes à doença mantendo sempre que possível, larga base genética e promovendo diversidade nos plantios (Ostry \& Mc Nabb, 1985). A região produtora de álamo no Brasil encontra-se em expansão e o sucesso deste empreendimento está vinculado a um correto manejo do patossistema, considerando o monitoramento da doença em função do clima, principalmente da temperatura. Assim, o manejo através da ampliação da base genética dos clones e o uso parcimonioso do controle químico permitirá equacionar o melhor controle deste patógeno a cada ano. Além disso, é importante o monitoramento da doença como indicativo da variação na virulência da população do patógeno frente aos clones utilizados ao longo dos anos de cultivo.

\section{REFERÊNCIAS BIBLIOGRÁFICAS}

BERGAMIN FILHO, A. \& AMORIM, L. Doenças de plantas tropicais: epidemiologia e controle econômico. São Paulo. Ceres. 1996.

BERGAMIN FILHO, A., CARNEIRO, S.M.T.P.G., GODOY, C.V., AMORIM, L., BERGER, R.D. \& HAU, B. Angular leaf spot 
on Phaseolus beans: Relationships between disease, healthy leaf area and yield. Phytopathology 87:506-515. 1997.

BERGER, R.D. The analysis of the effects of control measures on the development of epidemics. In: Kranz, J. \& Rotem, J. (Eds.) Experimental techniques in plant disease epidemiology. Heidelberg. Springer-Verlag. 1988. pp. 137-151.

BISSONNETTE, S.M., D’ARCY, C.J. \& PEDERSEN, W.L. Yield loss in two spring oat cultivars due to Puccinia coronata f. sp. avenae in the presence or absence of barley dwarf virus. Phytopathology 84:363-371. 1994.

CARNEIRO, S.M.T.P.G., AMORIM, L. \& BERGAMIN FILHO, A.Avaliação de dano provocado pela mancha angular em feijoeiro: relação entre severidade, área foliar e componentes de produção. Fitopatologia Brasileira 22:427-431. 1997.

CHASTAGNER, G.A. Poplar diseases. In: Jones, R.K. \& Benson, D.M. (Eds.) Diseases of woody ornamentals and trees in nurseries. St. Paul MN. APS Press. 2001. pp. 308-309.

GIORCELLI, A. \& VIETTO, L. Valutazione delléfficacia di alcunio fungicidi versu le ruggini del pioppo. Informatore Fitopatologico 45:59-62. 1995. (Abstract)

HAMELIN, R.C., SHAIN, L., FERRISS, R.S. \& THIELGES, B.A. Quantification of disease progress and defoliation in poplar leaf rust-eastern cottonwood pathosystem. Phytopathology 83:140-144. 1993.

MAY-DE MIO, L.L. \& AMORIM, L. Influência da temperatura e da duração do molhamento foliar nos componentes monocíclicos da ferrugem do álamo. Summa Phytopathologica 28:33-40. 2002.

MAY-DEMIO,L.L.,AMORIM,L.\&SCHUTA,L.R. Suscetibilidade de clones de Álamo à ferrugem no viveiro, eficiência de fungicidas e avaliação de danos. Summa Phytopathologica 28:46-51. 2002.

McCRACKEN, F.I., SCHIPPER, A.L. \& WIDIN, K.D. Observation on occurrence of cottonwood leaf rust in central United States. European Journal of Forest Pathology 14:226-233. 1984.

MOLTZAN, B.D. Melampsora leaf rust of Populus: its occurrence, natural distribution and a standardized scale for assessing infection types. (Master Thesis). Fargo ND. University of North Dakota. 1991.

NEWCOMBE, G., BRADSHAW JÚNIOR, H.D., CHASTAGNER, G.A. \& STETTLER, R.F. The major gene for resistance to Melampsora medusae f.sp. deltoidae in a hybrid poplar pedigree. Phytopathology 86:87-94. 1996.
NEWCOMBE, G. \& CHASTAGNER, G.A. A leaf rust epidemic of hybrid poplar along the lower Columbia River caused by Melampsora medusae. Plant Disease 77:528-531. 1993.

NOWACKI, M.J. \& FONTOURA, O.S. Alguns aspectos fitossanitários da silvicultura no Estado do Paraná. Anais, 1ำ Congresso Florestal Brasileiro, Curitiba, PR. 1968. pp. 315-316.

OSTRY, M.E. \& MC NABB, H.S. Susceptibility of Populus species and hybrids to disease in the north central United States. Plant Disease 69: 755-757. 1985.

PANDEY, P.C., SINGH, A., KARNATAK, D.C. \& BHARTARI, B.K. Melampsora laricis-populina on poplars in India and its control in nursery. The Indian Forester 122:1062-1067. 1996.

PATAKY, J.K., HEADRICK, J.M. \& SUPARYONO, L. Classification of sweet corn hybrid reactions to common rust, northern leaf blight, Stewart's wilt, and Goss' wilt and associated yield reductions. Phytopathology 78:172-178. 1988.

SCHIPPER, A.L. \& DAWSON, D.H. Poplar leaf rust - A problem in maximum wood fiber production. Plant Disease Reporter 58:721-723. 1974.

SPIERS, A.G. Species of Melampsora infecting conifers in New Zealand. Plant Disease Reporter 59:486-488. 1975.

STEENACHERS, J., STEENACHERS, M. \& STEENACHERS, V. Maladies des peupliers - Consequences sur la croissance et la qualite du bois. Les cahiers techniques de l'objetif 1. Bulletin trimestriel 1:4-20. 1995.

VIÉGAS, A.P. Alguns fungos do Brasil. IV. Uredinales. Bragantia 5:6-7. 1945.

WAGGONER, P.E. \& BERGER, R.D. Defoliation, disease and growth. Phytopathology 77:393-398. 1987.

WALKER, J. Melampsora medusae. CMI Descriptions of Pathogenic Fungi and Bacteria. No. 408. Kew. 1975.

WATSON, D.J. Comparative physiological studies on the growth of field crops. I. Variation in net assimilation rate and leaf area between species and varieties, and within and between years. Annals of Botany 11:41-76. 1947.

WIDIN, K.D. \& SCHIPPER, A.L. Epidemiology and impact of Melampsora medusae leaf rust on hybrid poplars. In: USDA FS, Intensive plantation culture - five year's research. General Technical Report NC-21. St. Paul MN. 1976. pp. 63-74. 